\title{
RADIOMETRIC CALIBRATION OF MULTI-WAVELENGTH AIRBORNE LASER SCANNING DATA
}

\author{
Christian Briese ${ }^{\mathrm{a}, \mathrm{b}} *$, Martin Pfennigbauer ${ }^{\mathrm{c}}$, Hubert Lehner ${ }^{\mathrm{a}}$, Andreas Ullrich $^{\mathrm{c}}$, W. Wagner ${ }^{\mathrm{a}}$, N. Pfeifer ${ }^{\mathrm{a}}$ \\ ${ }^{a}$ Institute of Photogrammetry and Remote Sensing of the Vienna University of Technology, Austria \\ ${ }^{\mathrm{b}}$ LBI for Archaeological Prospection and Virtual Archaeology, Vienna, Austria \\ ${ }^{c}$ RIEGL Laser Measurement Systems GmbH, Horn, Austria
}

Commission VII, WG VII/7

KEY WORDS: Airborne, Laser scanning, LIDAR, Mapping, Radiometry, Calibration

\begin{abstract}
:
Airborne laser scanning (ALS) is a widely used technique for the sampling of the earth's surface. Nowadays a wide range of ALS sensor systems with different technical specifications can be found. One parameter is the laser wavelength which leads to a sensitivity for the wavelength dependent backscatter characteristic of sensed surfaces. Current ALS sensors usually record next to the geometric information additional information on the recorded signal strength of each echo. In order to utilize this information for the study of the backscatter characteristic of the sensed surface, radiometric calibration is essential. This paper focuses on the radiometric calibration of multi-wavelength ALS data and is based on previous work on the topic of radiometric calibration of monochromatic (single-wavelength) ALS data. After a short introduction the theory and whole workflow for calibrating ALS data radiometrically based on in-situ reference surfaces is presented. Furthermore, it is demonstrated that this approach for the monochromatic calibration can be used for each channel of multi-wavelength ALS data. The resulting active multi-channel radiometric image does not have any shadows and from a geometric viewpoint the position of the objects on top of the terrain surface is not altered (the result is a multi-channel true orthophoto). Within this paper the approach is demonstrated by three different single-wavelength ALS data acquisition campaigns $(532 \mathrm{~nm}, 1064 \mathrm{~nm}$ and $1550 \mathrm{~nm}$ ) covering the area of the city Horn (Austria). The results and practical issues are discussed.
\end{abstract}

\section{INTRODUCTION}

Airborne laser scanning (ALS resp. airborne LIDAR) has established itself as a widely used technique for obtaining the geometry of the earth's surface. Next to the widely used geometric information, ALS systems typically provide additional information about the recorded signal strength of each echo. In order to utilize this information for the study of the backscatter characteristic of the sensed surface, radiometric calibration is essential (Höfle and Pfeifer, 2007). Relative radiometric calibration that tries to minimise radiometric differences within a strip and its neighbouring strips can be distinguished from absolute radiometric calibration where radiometric in-situ measurements (acting as control elements) are essential (Briese et al., 2008; Kaasalainen et al., 2009). In order to perform an absolute radiometric calibration the ALS observations that are affected by several influencing factors (e.g. range, atmosphere, incidence angle, etc.) have to be transformed into mission independent physical parameters. Nowadays, a wide range of ALS sensor systems can be found. They differ in the typical measurement range, measurement rate, the instrument size, and the echo determination possibilities (discrete echo vs. full-waveform recording). Furthermore, dependent on the different application areas (topography, hydrography, or glaciography), different laser wavelengths are utilised (Pfennigbauer and Ullrich, 2011) and the selected wavelength leads to a certain wavelength dependent backscatter characteristic of the sensed surface.

\footnotetext{
* Corresponding author, cb@ipf.tuwien.ac.at.
}

This paper focuses on the radiometric calibration of multiwavelength ALS data and is based on previous step-by-step extended research on the topic of radiometric calibration of monochromatic (single-wavelength) ALS data (cf. Wagner et al., 2006; Briese et al., 2008; Lehner and Briese, 2010; Roncat et al., 2011 and Lehner et al., 2011). In the following section the basic theory and practical workflow for the calibration of monochromatic ALS data based on in-situ reference surfaces is presented. Subsequently, it is demonstrated that this approach for the practical mission independent calibration can be used for each channel of multi-wavelength ALS data. Based on the calibrated backscatter per echo a radiometric image for each ALS wavelength can be calculated. In order to practically investigate multi-wavelength radiometric calibration, ALS data from three different single-wavelength ALS sensors (utilizing $1550 \mathrm{~nm}, 1064 \mathrm{~nm}$, and $532 \mathrm{~nm}$ lasers) covering the area of the city Horn (Austria) was acquired (cf. section 3). Next to the ALS data acquisition, in-situ radiometric field measurements were realised in order to allow an absolute radiometric calibration (cf. section 3.4). Section 4 provides next to the study of the three individual monochromatic calibration results the calibrated multi-wavelength reflectance image of the city of Horn. Finally, section 5 provides a short conclusion.

\section{RADIOMETRIC CALIBRATION}

Physical basis for the proposed radiometric monochromatic calibration of ALS data is the radar equation (Jelalian, 1992), the sought quantity is the calibration constant. Its calculation is 
presented in Section 2.1 the practical workflow for absolute radiometric calibration based on in-situ reference is given in 2.2 .

\subsection{Theory}

The relation of the transmitted laser power $P_{t}$ and the detected power of its echo $P_{r}$ for extended targets $i$ is given by the radar equation (Jelalian, 1992, Wagner, 2010):

$$
P_{r}=\frac{P_{t} D_{r}^{2}}{16 R_{i}^{2}} \eta_{a t m} \eta_{s y s} \gamma_{i}
$$

with the diameter of the receiver aperture $D_{r}$, the range between sensor and target $R_{i}$, the backscattering coefficient of the surface $\gamma_{i}$ as well as transmission factors for the atmosphere $\eta_{\text {atm }}$ and the ALS system $\eta_{s y s}$ (Wagner, 2010; Lehner et al., 2011). The backscattering coefficient $\gamma$ is normalised to the laser's transverse area and thus independent of $\mathrm{R}$ and the beam divergence of the ALS system. Assuming that all targets $i$ are diffuse (due to the typically short ALS wavelength for most surfaces justifiable) and reflecting according to the Lambertian law, the diffuse backscattering coefficient $\gamma_{d}$ depends only on the diffuse reflectance $\rho_{d}$ of the target surface and the angle of incidence $\theta$ (Lehner, 2011):

$$
\gamma_{d, i}=4 \rho_{d, i} \cos \theta_{i}
$$

In order to determine the backscattering coefficient $\gamma$ or subsequently the diffuse reflectance measure $\rho_{d}$ for each observed ALS target surface the unknown factors in equation (1) have to be estimated. The amount of unknown factors depends on the knowledge of the atmospheric conditions and the sensor specific available observations. While in the case of a modern full-waveform ALS system $P_{t}$ and $P_{r}$ can be determined in the post-processing of the digitised full-waveform signal, information about $D_{r}$ and $\eta_{s y s}$ is typically not available. In order to solve equation (1) for all echoes, the unknown parameters can be combined in the so-called calibration constant $\mathrm{C}_{\mathrm{cal}}$ and the combined value can be derived with the help of in-situ reference targets. This strategy also works for discrete return ALS systems where $P_{t}$ usually is unknown and/or in projects where no knowledge about the atmospheric conditions is available. The only precondition is that all combined values in $\mathrm{C}_{\mathrm{cal}}$ can be assumed to be constant for a certain flight mission. Depending on the knowledge of the ALS sensor and the flight mission $\mathrm{C}_{\mathrm{cal}}$ might combine the following parameters:

$$
C_{c a l}=\frac{16}{P_{t} D_{r}^{2} \eta_{s y s} \eta_{a t m}}
$$

\subsection{Practical radiometric calibration workflow}

As mentioned in the previous subsection, depending on the knowledge of the ALS system and the ALS mission the amount of observables and unknowns might vary. In the following the standard processing workflow for the radiometric calibration of full-waveform ALS data is presented step-by-step. Due to missing knowledge, some of the steps might not be executable for a certain dataset. This increases the summarised unknowns in $\mathrm{C}_{\mathrm{cal}}$ and leads to a value that might depend on the actual ALS mission (e.g. $P_{t}$ and $\eta_{a t m}$ ). However, using an in-situ reference target leads to an absolute calibration and inter-mission comparable results. Further differentiating the unknowns in $\mathrm{C}_{\text {cal }}$ and increasing the number of reference measurements tends to provide more accurate results.
The practical workflow based on full-waveform ALS data consists of the following steps:

1. Selection of the in-situ reference targets based on the ALS flight plan

2. Determination of the incidence angle dependent reflectance $\rho_{d}$ of the reference surfaces utilising a spectrometer or reflectometer (cf. Briese et al., 2008) that operates at the same ALS wavelength

3. Recording of meteorological data (aerosol type, visibility, water vapour, etc. for the estimation of an atmospheric model) during the flight mission in order to estimate the atmospheric transmission factor

4. Full-waveform decomposition (echo extraction and estimation of echo parameters)

5. Direct georeferencing of the ALS echoes and maybe strip adjustment in order to get an advanced relative and absolute georeferencing of the ALS data

6. Estimation of the local surface normal in order to consider the local incidence angle $\theta$

7. Estimation of $\mathrm{C}_{\mathrm{cal}}$ based on the ALS echoes within the insitu reference targets (e.g. defined by a polygon area)

8. Radiometric calibration of all echoes based on the determined value of $\mathrm{C}_{\text {cal }}$

At the end of this workflow, that can be realised with the program package OPALS (Opals, 2012), each ALS echo has assigned the additional calibrated diffuse reflectance measure that can be used in the further radiometric analysis of the ALS data. For the radiometric study of multi-channel ALS the above workflow has to be repeated for each channel and in-situ reference targets for all ALS wavelengths have to be determined.

Based on the radiometric attributes per echo a regular 2D raster can be interpolated (e.g. based on the first or last echo data). The result is an active radiometric image of the respective monochromatic ALS wavelength. The individual image pixel represents physical quantities that are independent of the illumination of the sun and ideally represent only the backscattering characteristics of the sensed (assumed extended target) surface. Multi-wavelength ALS images can be created by the combination of the individual monochromatic bands to multi-channel images.

\section{DATA SETS}

Currently, due to several technical and commercial restrictions, no compact multi-wavelength ALS system is commercially available (cf. Pfennigbauer and Ullrich, 2011). Therefore, in order to practically test the suggested procedure for the radiometric calibration of multi-channel ALS data, three different ALS sensor systems were utilised: RIEGL VQ-820-G, RIEGL VQ-580, and RIEGL LMS-Q680i operating at laser wavelengths of $532 \mathrm{~nm}, 1064 \mathrm{~nm}$, and $1550 \mathrm{~nm}$, respectively (for further instrument details, see RIEGL, 2012). Due to the limited mounting capabilities of the available aircrafts and the sensor availability, data was acquired within several independent flight missions. All three flights were performed based on the same flight plan. The selected study area is the city area of Horn (Austria) with its rural surrounding. In the following subsections a brief description of the individual flight missions and the in-situ reference measurements is provided. Figure 1 provides the boundaries of each acquired ALS strip colour coded per ALS system and furthermore gives an overview about the areas with in-situ reference surfaces. 
For all flights echo digitization with subsequent full-waveform decomposition or online waveform processing, determination of the echo attributes (amplitude and echo width of the emitted pulse and the received echo) as well as the direct georeferencing and strip adjustment was performed with RIEGL ALS software products (RIEGL, 2012).

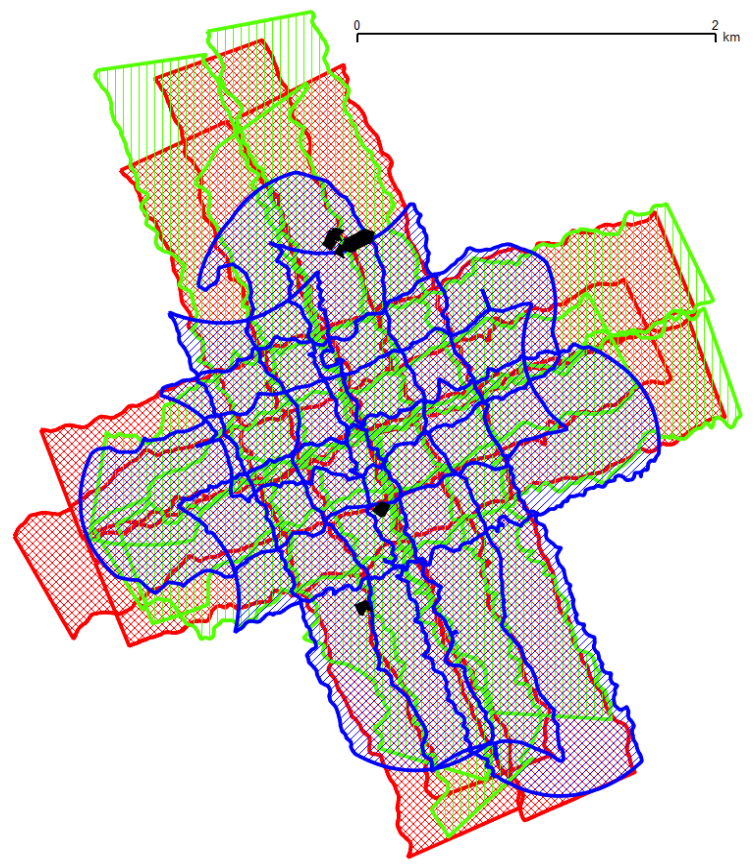

Figure 1. 2D overview of the study area Horn (Austria); boundaries of the ALS strips: RIEGL VQ-820-G (blue); RIEGL VQ-580 (green); RIEGL LMS-Q680i (red); areas with in-situ reference surfaces (black).

\subsection{Airborne Laser Scanning Missions}

Flight 1: RIEGL VQ-820-G: The flight with the RIEGL VQ820-G (532nm) sensor was performed on the $31^{\text {st }}$ of August 2011. All in all 6 flight strips were flown in two main directions and crossing over the city centre of Horn. The median point density (last echo) of this flight was 9.3 points $/ \mathrm{m}^{2}$. Due to an optimised scan pattern for hydrographical data acquisition (see Riegl, 2012) the begin and end of each ALS strip is, in contrast to the strip boundaries of the two other ALS systems, a curved boundary line section (cf. blue boundaries in Fig. 1).

Flight 2: RIEGL LMS-Q680i: The flight with the RIEGL LMS-Q680i $(1550 \mathrm{~nm})$ instrument was performed on the $22^{\text {nd }}$ of September 2011. A similar strip layout over the city of Horn was chosen (cf. Fig. 1). Median point density (last echo) of this flight was 11.8 points $/ \mathrm{m}^{2}$.

Flight 3: RIEGL VQ-580: The data with the third ALS sensor RIEGL VQ-580 (1064nm) was acquired on $4^{\text {th }}$ December 2011. The strip layout corresponds to the previous flight missions (see Fig. 1). The median point density (last echo) of this flight was 3.8 points $/ \mathrm{m}^{2}$.

\subsection{In-situ radiometric measurements}

The in-situ radiometric field measurements of reference surfaces were performed on the $5^{\text {th }}$ December 2011 (see black areas in Fig. 1). All in all 4 different reflecting surface types were chosen (see Table 1). They were all measured under dry condition (no visible water on top of the solid surfaces). All areas were measured multiple times (on slightly different locations) at zero angle of incidence (observation of the surface in normal direction) and the resulting median was selected as representative reflectivity value. For the following processing it is assumed that the reflectance of these reference surfaces follows the rule of Lambert.

The measurements for $1064 \mathrm{~nm}$ and $1550 \mathrm{~nm}$ were performed with two Riegl reflectometer instruments (see Briese et al., 2008), while for the measurements of the reflectance at $532 \mathrm{~nm}$ the spectrometer GER 1500 of the company Spectra Vision Corporation (SVC, 2012) was utilised.

\begin{tabular}{|c|l|c|}
\hline ID & \multicolumn{1}{|c|}{ Surface type } & $\begin{array}{c}\text { Reflectivity } \rho_{\mathrm{d}} \text { for } \\
532 / 1064 / 1550 \mathrm{~nm}\end{array}$ \\
\hline STP1 & Stone (pavement) & $0.1590 / 0.2282 / 0.2538$ \\
STP2 & Asphalt (road) & $0.1247 / 0.1958 / 0.2358$ \\
STP3 & Red stone (pavement) & $0.1183 / 0.3025 / 0.3612$ \\
STP4 & Asphalt (road) & $0.1004 / 0.2303 / 0.2624$ \\
\hline
\end{tabular}

Table 1 . In-situ measured reflectivity for $532 \mathrm{~nm}, 1065 \mathrm{~nm}$ and

$1550 \mathrm{~nm}$ at an incidence angle $\theta$ of zero (observation of the surface in normal direction) for the selected reference targets.

\section{RESULTS AND DISCUSSION}

The aim of the paper is the radiometric calibration of multiwavelength ALS data. Based on the calibrated multi-wavelength ALS data a radiometric multi-channel image can be determined. For this aim in a first step, based on the presented theory (subsection 2.1) and the proposed practical workflow (subsection 2.2), the ALS data of each flight is separately calibrated with the software package OPALS (OPALS, 2012).

In the following three subsections, the radiometric calibration results of the three flight missions are presented. Due to the different time gap between the ALS data acquisition and the insitu field measurements the polygons that represent the reference targets had to be adapted per flight mission in order to overcome problems with temporal changing objects on the reference surfaces (e.g. parking or driving cars).

The presented workflow leads to an absolute radiometrically calibrated monochromatic ALS data set per flight mission with assigned physical reflectance values (backscatter cross section, backscatter coefficient and diffuse reflectance measure) per echo. These additional quantities allow a monochromatic radiometric interpretation of the observed target. For the subsequent analysis and visualisation the diffuse reflectance measure per echo was selected. Furthermore, the aim of the paper is the determination of multi-wavelength radiometric quantities based on ALS observations. This can be achieved by the combination of different monochromatic reflectance images. This process and the results for the study site Horn are presented in subsection 4.4 .

\subsection{Radiometric Calibration of Flight 1: RIEGL VQ-820-G}

The processing for the radiometric calibration of the $532 \mathrm{~nm}$ ALS data was performed according to the workflow of subsection 2.2. The resulting reflectance values compared to the 
stored echo amplitude information can be inspected in figure 2. Furthermore, figure 3 provides a histogram of the resulting calibrated reflectance values. Due to a sensor specific behaviour the amplitudes are too low in close proximity to the strip boundary (Eq. 1 is too simple for large scan angles). Therefore, the resulting reflectance image was limited to a smaller area across the flight direction. All in all, this radiometric image is quite dark (median: 0.07) with maximum reflectance values of slightly above 0.25 .

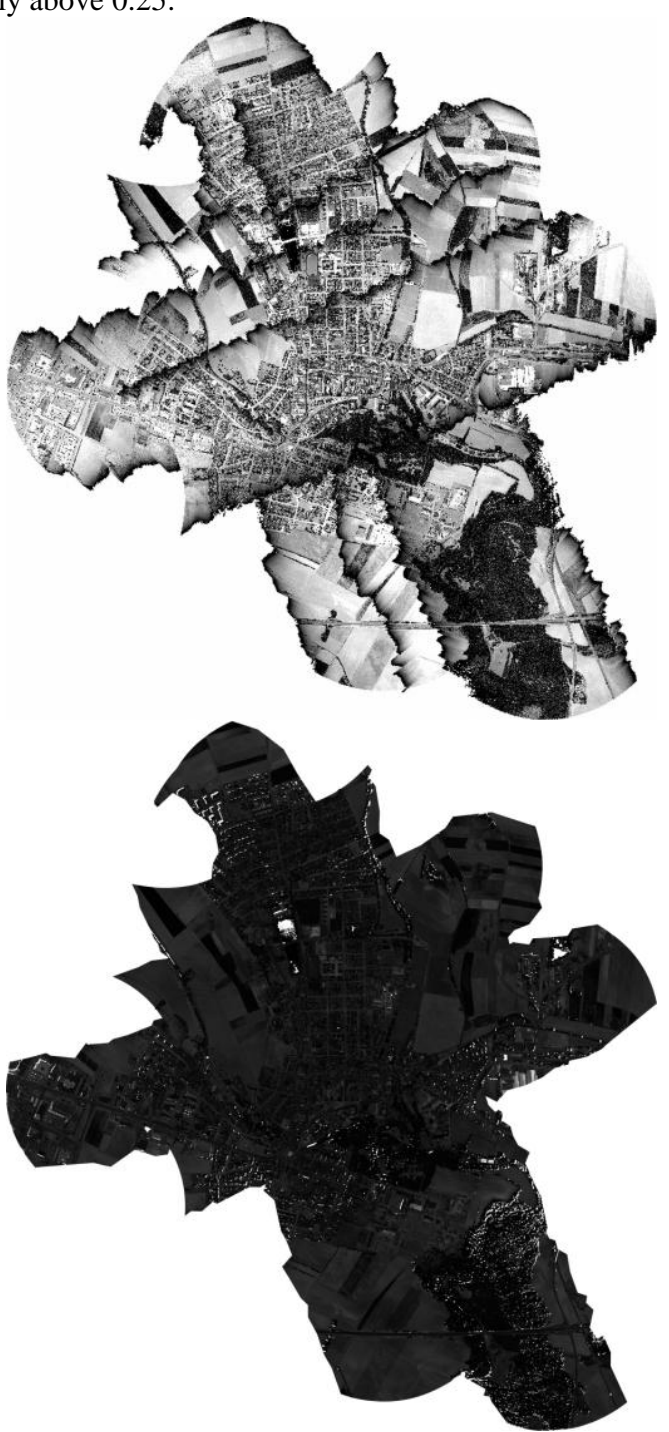

Figure 2. Upper image: amplitude image of the 532nm ALS data of the study area (linear scale from 0 (black) to 1000 (white)); Lower image: Calibrated radiometric reflectance at $532 \mathrm{~nm}$ (linear scale from 0 (black) to 1 (white)).

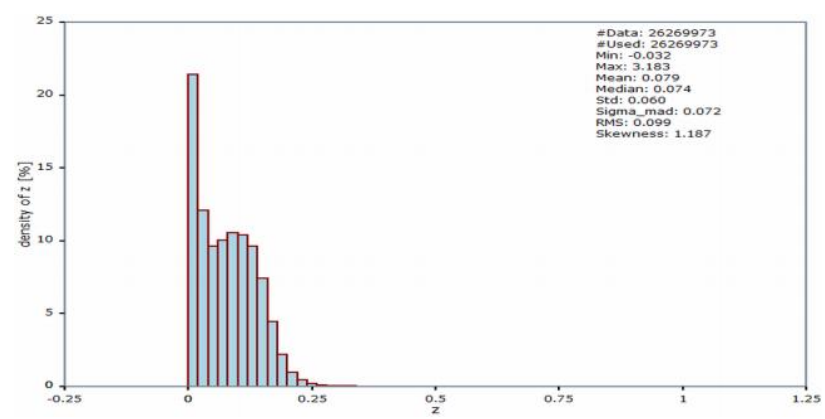

Figure 3. Histogram of the calibrated radiometric reflectance values displayed in the lower part of figure 2 .

\subsection{Radiometric Calibration of Flight 2: RIEGL LMS- Q680i}

Flight 2 (1550nm ALS data) was processed with the adapted polygons of the reference surfaces in the same manner than flight 1 . The resulting reflectance values and the histogram of the resulting calibrated reflectance values can be inspected in figure 4 . In contrast to the $532 \mathrm{~nm}$ reflectance visualisation in figure 2 , the image is significantly brighter which is also clearly visible by the comparison of both histograms. Most reflectance values ate in-between 0 and 0.75 , the median is at a reflectance of 0.33 .

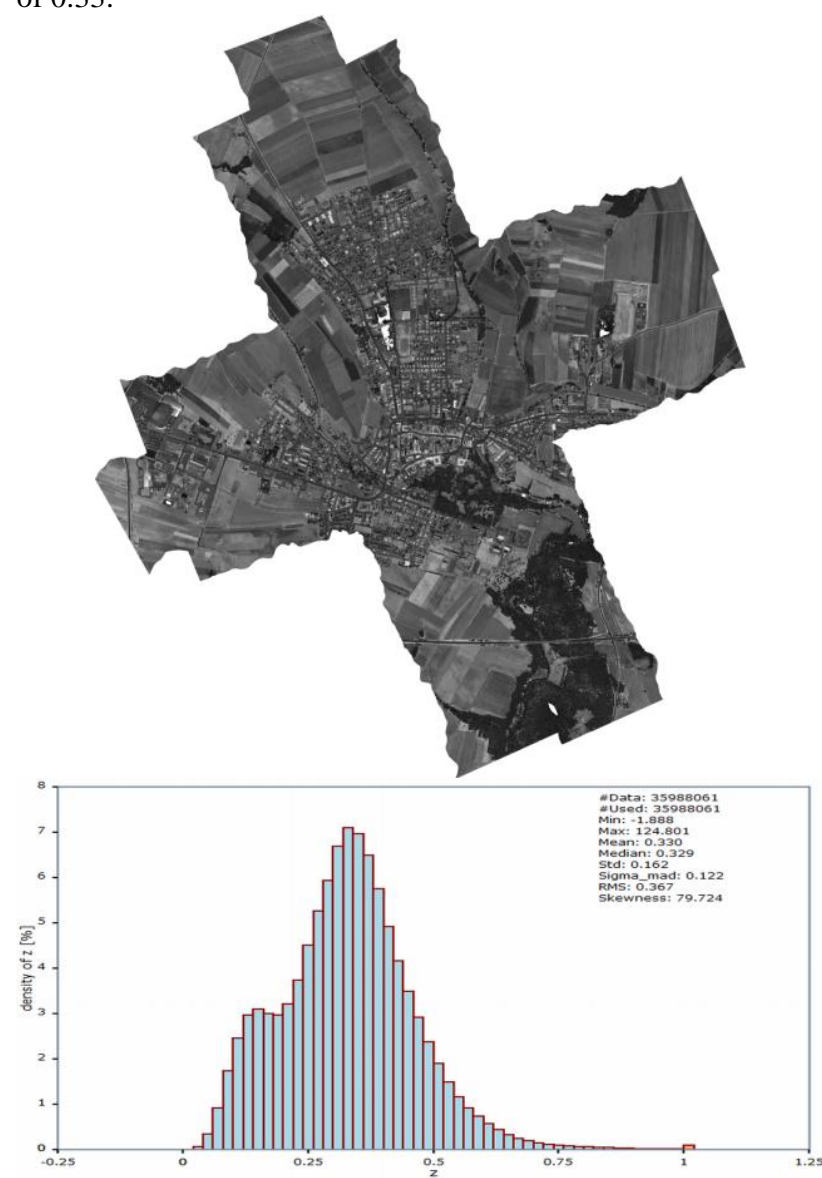

Figure 4. Upper image: Calibrated radiometric reflectance at 1550nm (linear scale from 0 (black) to 1 (white)); Lower image:

Histogram of calibrated radiometric reflectance values.

\subsection{Radiometric Calibration of Flight 3: RIEGL VQ-580}

With the further usage of OPALS the same radiometric calibration procedure was applied to the ALS data of flight 3 $(1064 \mathrm{~nm})$. The resulting calibrated reflectance values together with the respective histogram can be found in figure 5 . Compared to the other histograms the result for the VQ-580 ALS system $(1064 \mathrm{~nm})$ is much brighter (median: 0.49) and even approx. $15 \%$ of the reflective values are bigger than 1.0.

The higher reflectance values, especially those higher than 1.0, might be the result of the rather wet begin of December 2011. Just on the $5^{\text {th }}$ of December were the field measurements were performed a short time gap of approx. 3 hours offered dry surface conditions. Wetness on December the $4^{\text {th }}$ might have led to significantly more specular reflecting targets, provoking calibrated reflectance values to exceed the diffuse reflectance maximum of 1 . 


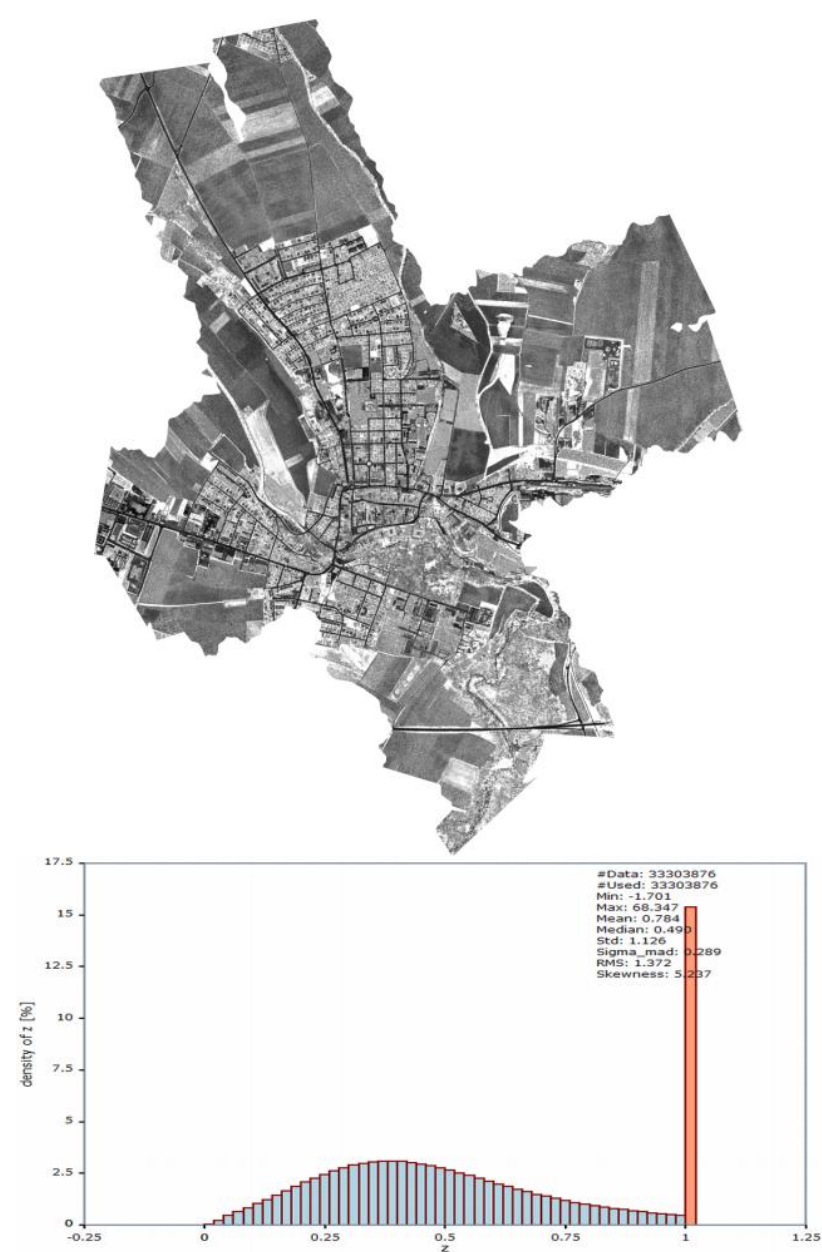

Figure 5. Upper image: Calibrated radiometric reflectance at $1064 \mathrm{~nm}$ (linear scale from 0 (black) to 1 (white)); Lower image:

Histogram of the calibrated radiometric reflectance values.

\subsection{Calibrated multi-wavelength reflectance}

Based on all three monochromatic radiometric reflectance results the calibrated multi-wavelength reflectance image can be generated. This active multi-channel image that can be directly visualised based on the results of the previous subsections. It can be inspected in Figure 6. Furthermore, in order to increase the brightness of the $532 \mathrm{~nm}$ channel figure 7 presents a different scaled visualisation. Additionally, figure 8 presents a detailed view of figure 7 of the city area of Horn.

It is important to note that these images are not influenced by any sun shadows (due to the active illumination of the sensed surface) and from a geometric viewpoint the position of the objects on top of the terrain surface is not altered (true orthophoto).

However, when assessing the quality of the radiometric multiwavelength image one has to have in mind that it is the result of three independent flight missions that were acquired within a time span of 96 days. For sure the surface conditions at the individual flight mission were not identical (see for example the high reflectance values in the $3^{\text {rd }}$ data acquisition campaign). Next to some temporal surface changes the influence of moisture (cf. Kaasalainen et al., 2010) has to be considered in the visual and analytic analysis.

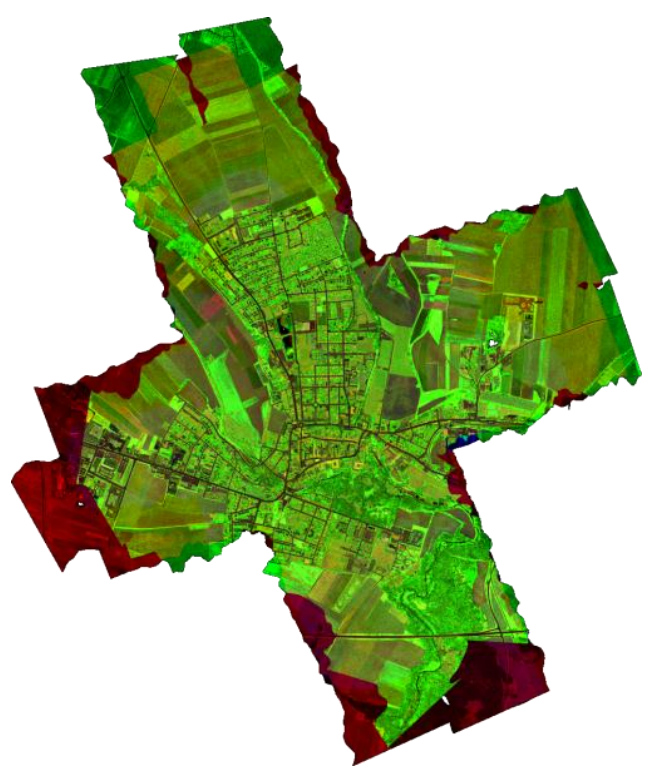

Figure 6. Active multi-wavelength reflectance image estimated from radiometrically calibrated ALS data sets; $532 \mathrm{~nm}$ (linear scale from 0 (black) to 1 (blue)); 1064nm (linear scale from 0 (black) to 1 (green)) and 1550nm (linear scale from 0 (black) to 1 (red)).

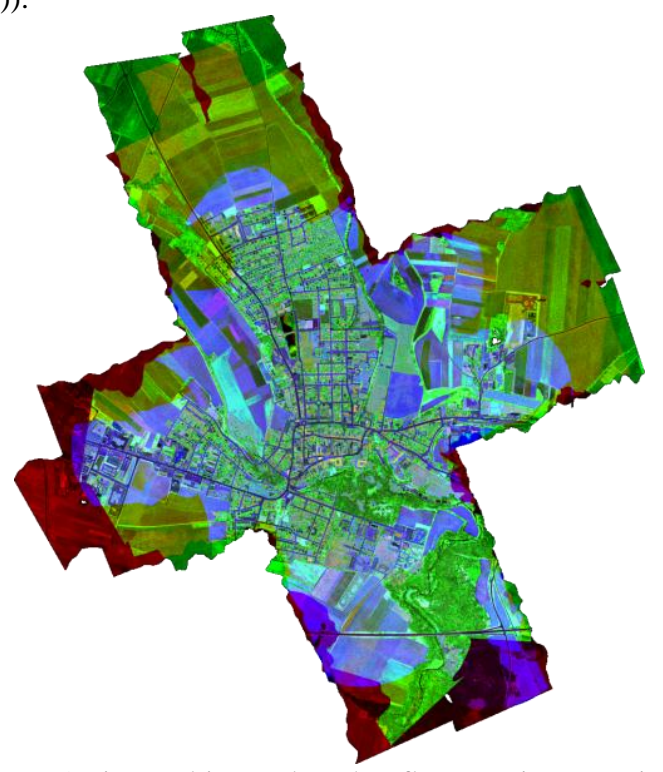

Figure 7. Active multi-wavelength reflectance image estimated from radiometrically calibrated ALS data sets; $532 \mathrm{~nm}$ (linear scale from 0 (black) to 0.15 (blue)); 1064nm (linear scale from 0 (black) to 1 (green)) and $1550 \mathrm{~nm}$ (linear scale from 0 (black) to 1 (red)).

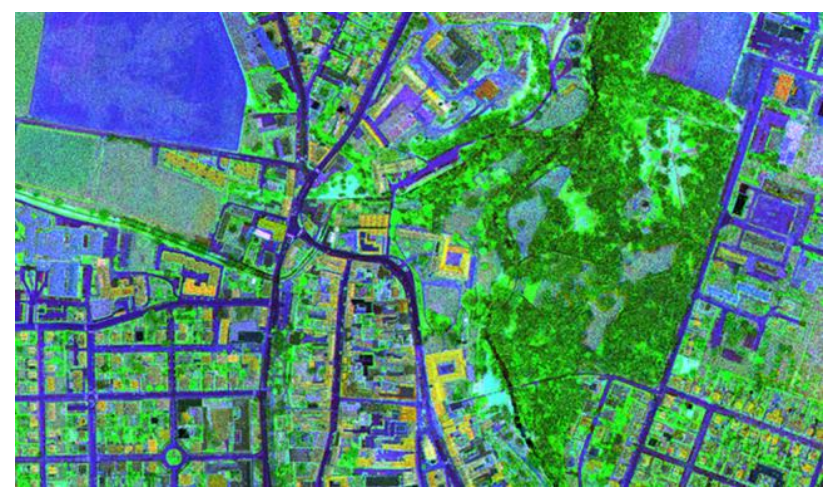

Figure 8. Detail view of figure 7 (rotated to the left). 


\section{CONCLUSION}

These first results show the applicability of calibrating multiwavelength radiometric imagery from ALS data and provide an insight into the challenges of radiometric processing and exploitation of multi-wavelength ALS data. Based on the resulting multi-wavelength reflectance information spectral analysis of the radiometric behaviour of the sensed surfaces at the three different wavelengths is possible. However, within the present multi-wavelength data set the three different data acquisition times have to be considered.

In the future a detailed quality analysis of the resulting reflectance values is essential. Next to improved data acquisition setups (contemporary data acquisition of the multiwavelength ALS data with similar sampling density) further studies on the analysis of several influence factors (atmosphere, sensor stability, etc.) have to be performed. This might lead to a refined model of the measurement process and might allow to increase the radiometric reliability and accuracy. Future research will be necessary to further analyse the practical application of calibrated active radiometric information from ALS data.

\section{ACKNOWLEDGEMENTS}

The Ludwig Boltzmann Institute for Archaeological Prospection and Virtual Archaeology (archpro.lbg.ac.at) is based on an international cooperation of the Ludwig Boltzmann Gesellschaft (A), the University of Vienna (A), the Vienna University of Technology (A), the Austrian Central Institute for Meteorology and Geodynamic (A), the office of the provincial government of Lower Austria (A), Airborne Technologies GmbH (A), RGZM-Roman- Germanic Central Museum Mainz (D), RÄ̈-Swedish National Heritage Board (S), IBM VISTAUniversity of Birmingham (GB) and NIKU-Norwegian Institute for Cultural Heritage Research (N). The authors thank Prof. Geert Verhoeven and Martin Wieser for the acquisition and processing of the spectrometer resp. reflectometer data.

\section{REFERENCES}

Briese, C., Höfle, B., Lehner, H., Wagner, W., Pfennigbauer, M., Ullrich, A., 2008. Calibration of full-waveform airborne laser scanning data for object classification. In: Turner, M.D., Kamerman, G.W. (Eds.), Proceedings of SPIE Laser Radar Technology and Applications XIII, vol. 6950, pp. 0H1-0H8.

Höfle, B., Pfeifer, N., 2007. Correction of laser scanning intensity data: Data and model-driven approaches. ISPRS Journal of Photogrammetry and Remote Sensing 62(6).

Jelalian, A. V., 1992. Laser Radar Systems. Artech House, Boston.

Kaasalainen, S., Hyyppa, H., Kukko, A., Litkey, P., Ahokas, E., Hyyppa, J., Lehner, H., Jaakkola, A., Suomalainen, J., Akujarvi, A., Kaasalainen, M., Pyysalo, U., 2009. Radiometric calibration of lidar intensity with commercially available reference targets. IEEE Transactions on Geoscience and Remote Sensing 47(2), pp. 588-598.

Kaasalainen, S., Niittymaki, H., Krooks, A., Koch, K., Kaartinen, H., Vain, A., Hyyppa, H., 2010, Effect of Target Moisture on Laser Scanner Intensity, IEEE Transactions on Geoscience and Remote Sensing 48(4), pp. 2128-2136.
Lehner, H., 2011. Radiometric calibration of airborne laser scanner data. Master thesis, Institut für Photogrammetrie und Fernerkundung der Technischen Universität Wien.

Lehner, H. and Briese, C., 2010. Radiometric calibration of fullwaveform airborne laser scanning data based on natural surfaces. In: ISPRS Technical Commission VII Symposium 2010: 100 Years ISPRS - Advancing Remote Sensing Science. International Archives of the Photogrammetry, Remote Sensing and Spatial Information Sciences 38 (Part 7B), Vienna, Austria, pp. 360- 365.

Lehner, H., Kager, H., Roncat, A., Zlinszky, A., 2011, Consideration of laser pulse fluctuations and automatic gain control in radiometric calibration of airborne laser scanning data, in: "Proceedings of 6th ISPRS Student Consortium and WG VI/5 Summer School", 6 pages.

OPALS, 2012. www.ipf.tuwien.ac.at/opals. Homepage of the software OPALS, accessed: January 2012.

Pfennigbauer, M., Ullrich, A., 2011. Multi-Wavelength Airborne Laser Scanning. ILMF 2011, New Orleans, February 7-9, 2011.

Riegl, 2011. www.riegl.com. Homepage of the company RIEGL Laser Measurement Systems GmbH, accessed: January 2012.

Roncat, A., Lehner, H., Briese, C., 2011, Laser Pulse Variations And Their Influence On Radiometric Calibration Of FullWaveform Laser Scanner Data, Talk: ISPRS Workshop Laser Scanning 2011, Calgary, Canada; 2011-08-29 - 2011-08-31, International Archives of the Photogrammetry, Remote Sensing and Spatial Information Sciences 38, (Part 5) / W12, ISSN: 1682-1777; 6 pages.

SVC, 2012. Spectrometer GER 1500 of the company Spectra Vista Corporation, http://www.spectravista.com/1500.html, accessed: January 2012.

Wagner, W., 2010. Radiometric calibration of small footprint full-waveform airborne laser scanner measurements: Basic physical concepts. ISPRS Journal of Photogrammetry and Remote Sensing 65 (6 (ISPRS Centenary Celebration Issue)), pp. 505-513. International Archives of Photogrammetry, Remote Sensing and Spatial Information Sciences 38, Part 7B, pp. 360-365.

Wagner, W., Ullrich, A., Ducic, V., Melzer, T. and Studnicka, N., 2006. Gaussian decomposition and calibration of a novel small-footprint full-waveform digitising airborne laser scanner. ISPRS Journal of Photogrammetry and Remote Sensing 60(2), pp. 100-112. 